\title{
Safety and therapeutic effect of mesenchymal stem cell infusion on moderate to severe ulcerative colitis
}

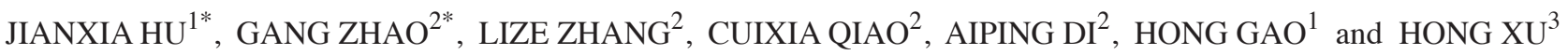 \\ ${ }^{1}$ Stem Cell Research Center; ${ }^{2}$ Anorectal Department, The Affiliated Hospital of Qingdao University, \\ Qingdao, Shandong 266003; ${ }^{3}$ Endoscopy Center, The First Affiliated Hospital of Jilin University, \\ Changchun, Jilin 130000 , P.R. China
}

Received June 24, 2015; Accepted August 11, 2016

DOI: $10.3892 /$ etm.2016.3724

\begin{abstract}
One of the primary targets of the clinical treatment of ulcerative colitis (UC) is to repair the damaged colonic mucosa. Mesenchymal stem cells (MSCs) have therapeutic potential in regenerative medicine due to their differentiation capacity and their secretion of numerous bioactive molecules. The present study describes a clinical trial (trial registration no. NCT01221428) investigating the safety and therapeutic effect of MSCs derived from human umbilical cord on moderate to severe UC. Thirty-four patients with UC were included in group I and treated with MSC infusion in addition to the base treatment, and thirty-six patients were in group II and treated with normal saline in addition to the base treatment. One month after therapy, 30/36 patients in group I showed good response, and diffuse and deep ulcer formation and severe inflammatory mucosa were improved markedly. During the follow up, the median Mayo score and histology score in group I were decreased while IBDQ scores were significantly improved compared with before treatment and group II $(\mathrm{P}<0.05)$. Compared with group II, there were no evident adverse reactions after MSC infusion in any of the patients in group I, and no chronic side effects or lingering effects appeared during the follow-up period. In conclusion, MSC infusion might be a useful and safe therapy for treating UC.
\end{abstract}

\section{Introduction}

Ulcerative colitis (UC) is a chronic inflammatory bowel disease affecting the mucosa and submucosa of the rectum, which may

Correspondence to: Dr Gang Zhao, Anorectal Department, The Affiliated Hospital of Qingdao University, 16 Jiangsu Road, Qingdao, Shandong 266003, P.R. China

E-mail: qdyxysxf@163.com

*Contributed equally

Key words: ulcerative colitis, mesenchymal stem cells, mucosal healing, immune regulation, cell therapy extend to the entire colon, and is characterized by a relapsing and remitting course (1). The symptoms of UC commonly include bloody diarrhea, rectal bleeding or rectal urgency. The severity of these symptoms is typically related to the extent of mucosal inflammation and can be observed by colonoscopy $(2,3)$. The induction of remission and mucosal healing are the goals of therapy. Aminosalicylates, azathioprine, 6-mercaptopurine and infliximab may be used for maintenance, however, the above treatments do not adequately work in all patients or are not well tolerated $(4,5)$. There remains an urgent need for novel therapeutic options to cure UC.

Mesenchymal stromal cells (MSCs) can be easily isolated and amplified from umbilical cord and other tissues. The characteristics of MSCs result in extensive use in numerous diseases, including tissue degeneration and refractory chronic inflammatory diseases (6). In UC, the inflammatory response is mediated by cytokines similar to the Th2 response and different immunocytes (7). The involvement of T-cells, natural killer (NK) cells and dendritic cells in UC pathophysiology has been confirmed in a large recent genome-wide association study (8). Recent data have indicated that MSCs can restore tissues by their immunomodulatory function, differentiation and paracrine effects. MSCs can modulate allogeneic immune cell responses by affecting dendritic cells, T-lymphocytes and NK cells (9). In addition, MSCs appear to regulate the immune reaction in inflamed tissues by affecting the formation and secretion of pro-inflammatory cytokines and chemokines, such as prostaglandin E2 and interleukin (IL)-6 (10). MSCs may induce a cytokine profile shift in the T helper (Th) 1/Th2 balance toward the anti-inflammatory phenotype $\mathrm{Th} 2$, which is accompanied by an increase of T regulatory lymphocytes and IL-10 $(11,12)$.

Regarding the treatment of $\mathrm{UC}$, it can be suggested that MSCs are effective by their immune modulatory properties coupled with a tissue repair capacity. Based on this consideration and current findings, a clinical trial (trial registration no. NCT01221428) was performed to investigate the safety and therapeutic effect of MSCs derived from human umbilical cord on moderate to severe UC.

\section{Materials and methods}

Study design. The present trial was a phase I/II, 24 month, randomized controlled study conducted in patients with 
moderate to severe UC. The study was conducted in accordance with the Declaration of Helsinki and approved by the Ethical Committee of the Affiliated Hospital of Qingdao University (Qingdao, China). Each patient provided written informed consent prior to entering the study. During the whole study, the investigators remained blind to the treatment administered.

Patients. Between September 2011 and December 2012, all patients were selected from The Affiliated Hospital of Qingdao University for the treatment of UC. UC was diagnosed according to current established clinical, endoscopic, radiologic and histologic guidelines (13). The extent of UC was evaluated based on the Mayo score classification $(14,15)$. Eligibility criteria for study entry included patients $\geq 18$ years of age, diagnosed with active UC with a Mayo score at baseline (range between 8 and 10). Exclusion criteria included infectious complications, toxic megacolon, previous colorectal surgery, history of colorectal dysplasia or any malignancy, pregnancy and any psychological illness.

Treatment. All patients were on stable doses of aminosalicylates for $\geq 4$ weeks prior to enrollment and all patients continued their individual regimens throughout the trial. Patients in group I were treated with MSC infusions twice besides the base treatment, one via injection into the veins in the back of the hand, and the other injected into the superior mesenteric artery by interventional catheterization, with a 7 day interval. Patients in group II were treated the same but with normal saline, which had the same volume and appearance as the MSC solution. The volumes of the parenteral solution of MSCs and normal saline for intravenous injection in the two groups were $50 \mathrm{ml}$ and the average cell number was $3.8 \pm 1.6 \times 10^{7}\left(0.5 \times 10^{6}\right.$ cells $/ \mathrm{kg}$; range, $2.3-4.7 \times 10^{7}$ cells $)$, and the volumes of the parenteral solution of MSCs and normal saline for mesenteric artery injection by interventional catheterization in the two groups were $10 \mathrm{ml}$ and the cell number was $1.5 \times 10^{7}$. To undergo MSC infusion, all patients were admitted to The Affiliated Hospital of Qingdao University. During the follow-up, the dosage of aminosalicylates administered were adjusted according to the patient's condition.

MSCs were provided by the Human Umbilical Cord Mesenchymal Stem Cell Bank (Qingdao, China). MSCs were cultured and expanded in a laminar flow laboratory (designed according to Good Medical Practice) for four passages to prepare stable final cell products that qualify for aerobe, mycoplasma, hepatitis B virus, hepatitis C virus, human immunodeficiency virus, Epstein-Barr virus, cytomegalovirus, syphilis and endotoxin testing. Cells were stained with CD-PE and CD-FITC (from the Human MSC Analysis kit; cat. no. 562245; BD Biosciences, Franklin Lakes, NJ, USA) and then analyzed by flow cytometry with a FACSCalibur flow cytometer (BD Biosciences). These cells highly expressed cluster of differentiation (CD) 90 (89.37\%), CD105 (82.26\%), CD73 (90.63\%), and CD146 (65\%) but not CD34 (0.23\%), CD45 (0.02\%) and Human Leukocyte Antigen-D Related (0.03\%) (Human MSC Analysis kit). The chromosomal karyotype of UC-MSC was determined as normal using a Metascan Karyotyping System (Imstar, Paris, France).
Clinical assessment and follow-up. The final evaluation prior to the first infusion of MSC was used as baseline for all analyses. Laboratory assessments of C-reactive protein (CRP), erythrocyte sedimentation rate (ESR), blood counts, liver enzymes, total protein, albumin, total cholesterol, triacylglycerol, serum urea nitrogen, creatinine and electrolytes were performed in the Clinical Laboratory of The Affiliated Hospital of Qingdao University. All samples were handled by the Clinical Laboratory of The Affiliated Hospital of Qingdao University. Full Mayo scores and Inflammatory Bowel Disease Questionnaire (IBDQ) scores $(16,17)$ were determined every 3 months in the first year after therapy, and then every 6 months during the second year of follow-up. The Mayo subscores for stool frequency and rectal bleeding were calculated based on entries from patient diaries using the worst diary entry from the 3 days before each study visit for each subscore. In all patients who had undergone follow-up endoscopy, it was assessed whether mucosal healing was achieved or not. Clinical response was defined as a decrease from baseline in the total Mayo score of $\geq 3$ points and $\geq 30 \%$, with an accompanying decrease in the subscore for rectal bleeding of at least 1 point or an absolute subscore for rectal bleeding of 0 or 1. Clinical remission was defined as a total Mayo score of $\leq 2$ points, with no individual subscore exceeding 1 point. Mucosal healing was defined as an absolute subscore for endoscopy of 0 or 1 . Tissue samples of colon were fixed in $4 \%$ formalin solution overnight and embedded in paraffin by the conventional method, then cut into $4 \mu \mathrm{m}$ thick sections. The sections were stained with hematoxylin and eosin for gross histological examination using a fluorescence microscope.

Study objectives. The primary study endpoint was safety and a clinical response documented by full Mayo scores and IBDQ scores at 3 months following treatment completion, and the secondary endpoints were safety and improvements of Mayo scores and IBDQ scores at 24 months.

Safety assessments. Patients were monitored continuously for adverse events, including evaluations every 2 weeks during the follow-up period. Other safety parameters (vital signs and clinical laboratory parameters) were ascertained monthly.

Data collection. A data collection form was developed according to the objectives of the present study. Training of researchers and research assistants was performed during a pilot data collection period and the case record form was standardized. Site visits by internal and external auditors were performed regularly to assure quality of the data and the clinical trial process.

Statistical analysis. All statistic analyses were performed using SPSS version 15.0 software (SPSS, Inc., Chicago, IL, USA). Data are presented as the mean \pm standard deviation. Differences between the means of the baseline values of group I and group II were analyzed using Student's t-test. Comparisons between time-dependent changes at the time of baseline and different time points following treatment were performed using repeated measure analysis of variance and post hoc analysis with the Bonferroni correction. $\mathrm{P}<0.05$ was considered to indicate a statistically significant difference. 


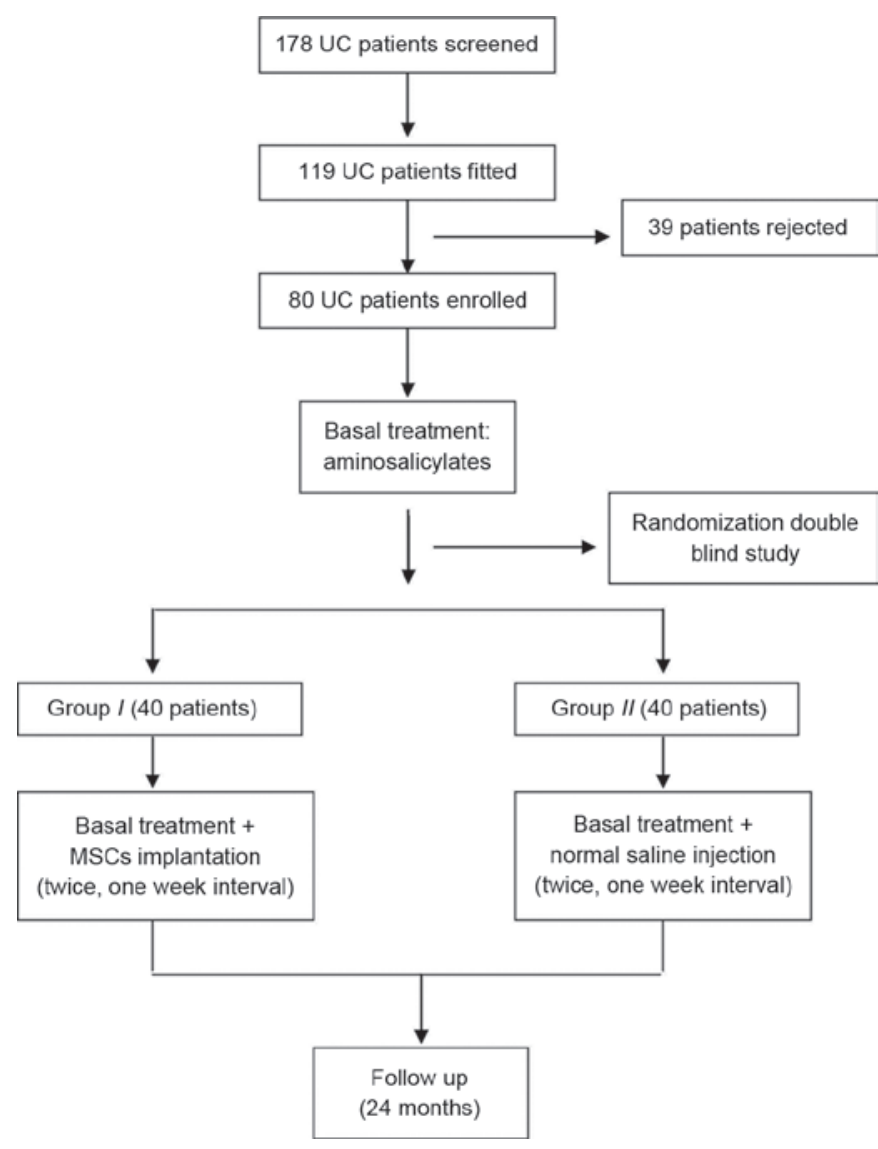

Figure 1. Procedure of the trial. UC, ulcerative colitis; MSC, mesenchymal stem cell.

\section{Results}

Patient characteristics. A total of 80 patients with UC were initially enrolled in the study (Fig. 1) and randomly divided into groups $I$ and $I I$. Four patients in group $I I$ and six patients in group $I$ withdrew from the study following therapy because of intolerance of the colonoscopy examination or lack of time to take part in the study. In total, 70 patients completed the entire study and their data were analyzed. Overall, there were 43 men and 27 women, with mean age of $42.7 \pm 9.6$ years (range, 18-52 years). The baseline characteristics of all patients are presented in Table I. There were no significant differences in clinical findings, laboratory examinations, Mayo scores or IBDQ scores between the two groups prior to the trial. A cancer screening test confirmed no cancer in all patients.

Safety evaluation. No clinical symptoms and no significant changes in vital signs were observed during and after cell therapy. Compared with group II, there were no evident adverse reactions following MSC infusion in any of the patients in group I, and no chronic side effects or lingering effects appeared during the follow-up period.

Efficacy. One month after therapy, 30/36 patients in group I showed good response, such as remission of stomachache, diarrhea and mucous bloody stool. All eligible patients underwent endoscopy at baseline and every follow-up point after therapy. As shown in Fig. 2, diffuse and deep ulcer formation and
Table I. Baseline patient characteristics.

\begin{tabular}{lcc}
\hline Characteristic & $\begin{array}{c}\text { Group I } \\
(\mathrm{n}=34)\end{array}$ & $\begin{array}{c}\text { Group II } \\
(\mathrm{n}=36)\end{array}$ \\
\hline Gender, male/female & $21 / 13$ & $22 / 14$ \\
Median age, year & $42.9 \pm 23.1$ & $43.7 \pm 28.7$ \\
Duration of disease, year & $5.6 \pm 4.2$ & $6.1 \pm 4.9$ \\
Extent of disease & & \\
Total colitis (\%) & $24(70.6)$ & $24(66.7)$ \\
Left-sided colitis $(\%)$ & $10(29.4)$ & $12(33.3)$ \\
Mayo score at baseline & $8.9 \pm 3.2$ & $8.5 \pm 3.8$ \\
IBDQ at baseline & $178.9 \pm 26.7$ & $183.1 \pm 32.9$ \\
CRP, mg/l & $35.96 \pm 15.75$ & $37.58 \pm 19.03$ \\
ESR, mm/h & $73 \pm 22.1$ & $69 \pm 18.2$ \\
\hline
\end{tabular}

Values are presented as the number of patients or mean \pm standard deviation. IBDQ, inflammatory bowel disease questionnaire; CRP, C-reactive protein; ESR, erythrocyte sedimentation rate.

severe inflammatory mucosa were observed prior to therapy, but the findings were greatly improved in group I following therapy.

Change in Mayo scores. Three months after therapy, the ratio of clinical responses ( $\geq 3$ point decrease in the Mayo score) or remission (Mayo score 0 or 1) was significantly higher in group I compared with group II (85.3 vs. $15.7 \%$; $\mathrm{P}=0.007$; Fig. 3A). The median Mayo score was improved in 27 patients in group I at the third month after cell therapy and reached the lowest level at six months, then sustained or showed a little fluctuation during the entire follow-up period. In group II, the median Mayo score of all patients fluctuated during the entire follow-up period (Fig. 3B).

Change in histological assessment scores. Histology scores from the colonic biopsies are presented in Fig. 4. The median histology score in group I decreased following cell therapy. Histological findings observed at baseline, including abruption of the surface layer, goblet cell depletion, crypt abscesses, gland collapse and inflammatory cell infiltration, improved following therapy. The median histology score in group I was significantly lower compared with that in group II $(\mathrm{P}<0.05$; Fig. 4).

Change in $I B D Q$ scores. Inflammatory Bowel Disease Questionnaire (IBDQ) scores were analyzed to evaluate any therapy-induced changes in health-related quality of life. Fig. 5 shows changes in total IBDQ scores. The IBDQ scores in group I were significantly improved compared with baseline (128.6 vs. $181.9 ; \mathrm{P}=0.002)$ at the third month of the trial, and were higher than group II from the sixth month of the trial. Although IBDQ scores steadily increased in group II throughout the trial, the change in scores failed to reach statistical significance by the end of the trial $(\mathrm{P}>0.05)$.

Change in cytokine levels and blood test. The mean plasma cytokine levels, including TNF- $\alpha$, IL-6 and IFN- $\gamma$, were not 

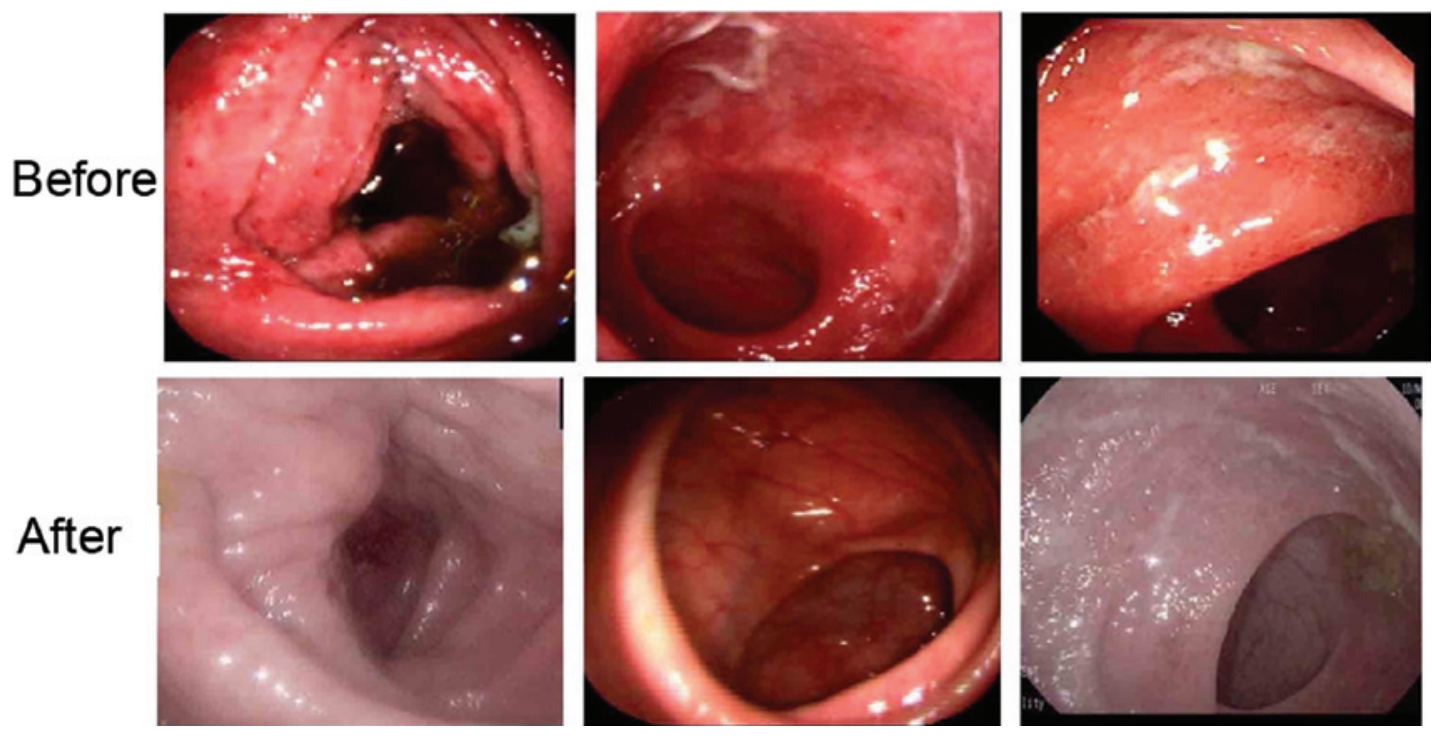

Figure 2. Endoscopic findings of patients in group I before and after therapy.

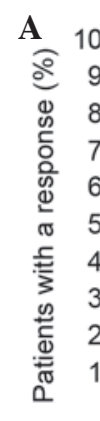

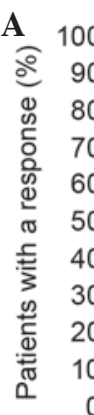

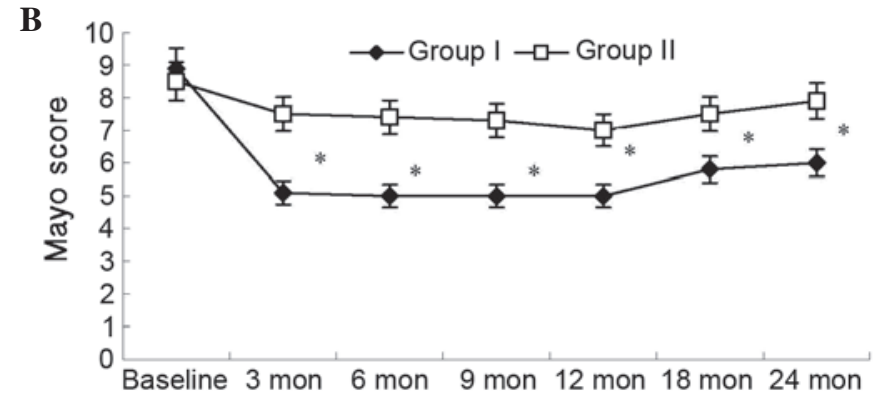

Figure 3. Ratio of clinical response and change of Mayo scores over time. (A) The ratio of clinical response ( $>3$ points decrease in Mayo score) in groups I ( $n=34)$ and II $(n=36)$. ${ }^{*} \mathrm{P}=0.007$ vs. group II. (B) The change of Mayo scores in groups I and II over time. ${ }^{*} \mathrm{P}<0.05$ vs. group II. Mon, months.

significantly different between the two groups at baseline and during the follow-up period.

The changes in results of blood test between the two groups was analyzed. In group I, CRP and ESR significantly decreased at the third month $(0.62$ to $0.18 \mathrm{mg} / \mathrm{l}, \mathrm{P}=0.03$; 76 to $23 \mathrm{~mm} / \mathrm{h}, \mathrm{P}=0.02$ ) compared with the baseline. There was no significantly difference between two groups. Data are presented in Fig. 6. There were no other significant changes and no adverse events related to blood count, hemoglobin and hematocrit, liver enzymes, total protein, albumin, total cholesterol, triacylglycerol, serum urea nitrogen, creatinine
A
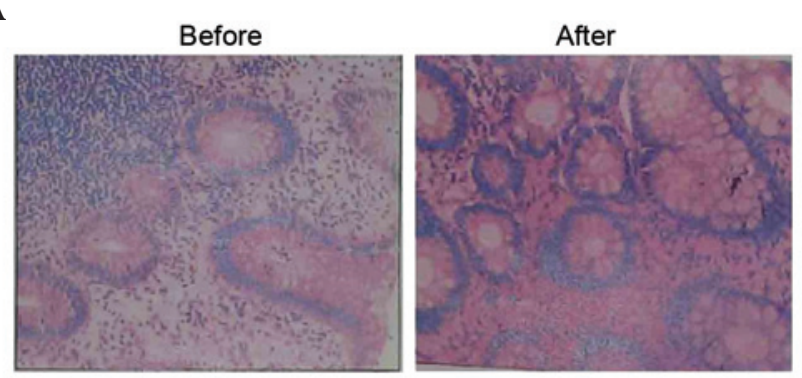

B

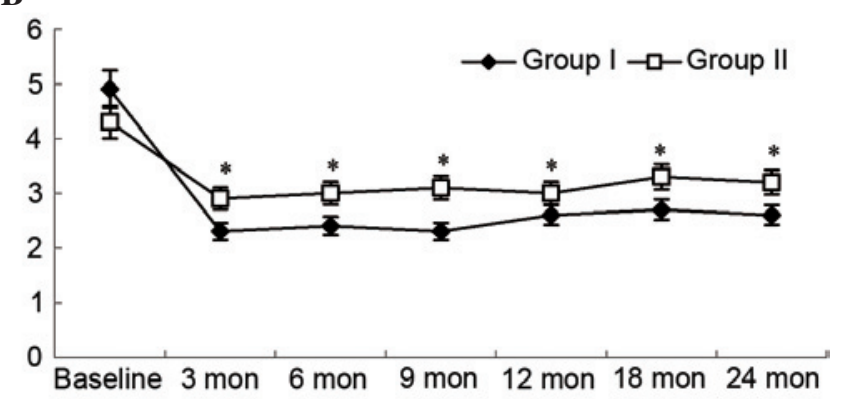

Figure 4. Pathological findings and change in histological scores over time (A) The change of pathological findings in patients of group I before and after therapy; magnification, x10. (B) The change of histological scores in the two groups over time. ${ }^{*} \mathrm{P}<0.05$ vs. group II. Mon, months.

and electrolytes in group I compared with group II (data not presented).

\section{Discussion}

One of the main targets of the clinical treatments for UC is to repair the damaged colonic mucosa. MSCs have great therapeutic potential in regenerative medicine due to their differentiation capacity and their secretion of numerous bioactive molecules (18). There are a number of studies focused on the attractive regenerative properties of MSCs, and evidence indicating that MSCs can promote regeneration of injured tissue (19-22). MSCs have low immunogenicity and display 


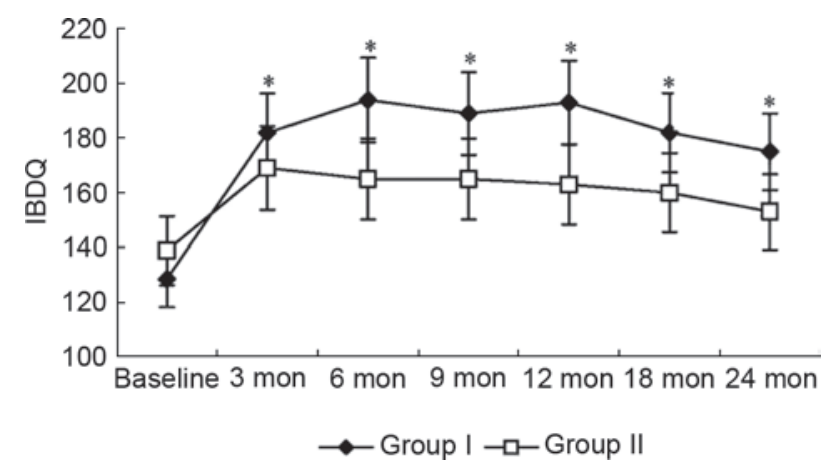

Figure 5. Change of IBDQ scores over time in groups I and II. "P<0.05 vs. group II. IBDQ, Inflammatory Bowel Disease Questionnaire score; mon, months.

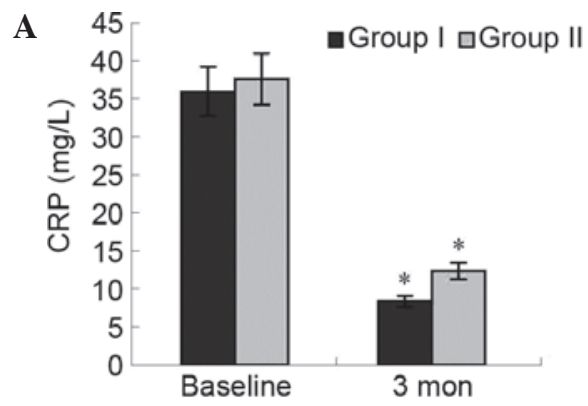

B

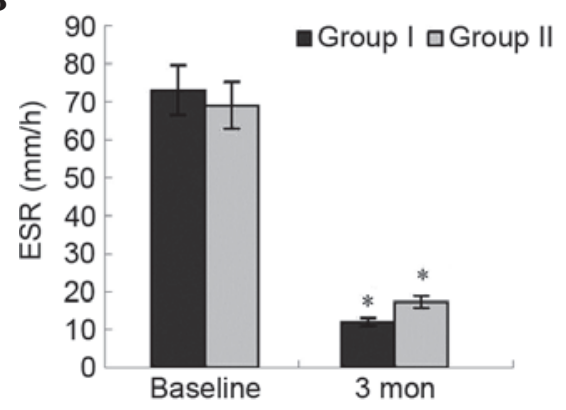

Figure 6. Change of (A) CRP and (B) ESR in groups I and II. Three months after therapy, the levels of CRP and ESR in the two groups significantly decreased at the third month compared with the baseline measurement; ${ }^{*} \mathrm{P}<0.05$ vs. group I baseline; ${ }^{*} \mathrm{P}<0.05$ vs. group II baseline. There was no significant difference between the two groups after therapy.

immunosuppressive proprieties (23), and can trigger the release of anti-inflammatory cytokines (24). Their capacity to suppress $\mathrm{T}$ cell activities and induce apoptosis provides a rationale for applying these cells in UC therapy $(25,26)$.

In the present clinical trial, the safety and therapeutic effect of MSCs derived from human umbilical cord were investigated on patients with moderate to severe UC and followed up for two years. The results showed that all evaluations, including endoscopic findings, pathological findings, Mayo scores and IBDQ scores, were markedly improved in group I following MSCs infusion compared with baseline and group II. These results indicate that MSC infusion is a safe and effective therapy for UC.

Mechanisms directing in vivo homing and engraftment of MSCs are unclear and depend on complex interactions between numerous signaling events. Several studies have demonstrated the ability of MSCs to preferentially migrate to sites of injury when infused in animal models (27-29). After receiving appropriate signals during tissue inflammation, MSCs could migrate to the lesions of the colon where they assisted in recovery, displaying high therapeutic potential with regards to tissue repair and/or the control of local inflammation (30). The expression of growth factors, cytokines and extracellular matrix receptors by MSCs may drive this process $(31,32)$. In the present study, the endoscopic findings, pathological findings and Mayo scores of patients in group I were markedly improved compared with group II, indicating that MSCs serve a role at the local sites.

MSCs can reduce colonic inflammation by downregulating the production of inflammatory mediators by mucosal immune cells, and by increasing the levels of the anti-inflammatory cytokines (33). In UC, the immunologic response is reflected by the imbalance in Th1 and Th2 cells, and thus the cytokine production at different stages of disease (34). Intravenous treatment with MSCs could increase the levels of the anti-inflammatory cytokines IL-10 and IL-4, and decrease the levels of the pro-inflammatory cytokine IL-6 $(35,36)$. Chatterjee et al (37) demonstrated that high levels of IFN- $\gamma$ produced by $\mathrm{T}$ cells in contact with MSCs resulted in the activation of the immunosuppressive effect of MSCs. Crucitti et al (38) assessed the therapeutic effect of MSCs by measuring inflammatory cytokines such as TNF- $\alpha$, IFN- $\gamma$, IL- 6 and IL- $1 \beta$, and chemokines, such as macrophage inhibitory protein II, which were significantly decreased in treated mice. Hengartner et al (39) assessed the MSC therapeutic effect by measuring the RNA expression of inflammatory mediators such as TNF- $\alpha$, IL- $1 \beta$, cyclo-oxygenase 2 , basic fibroblast growth factor, hepatocyte growth factor and vascular endothelial growth factor, all of which significantly decreased in MSC-treated mice. In a study by Forte et al (40), human umbilical cord MSCs were used to treat acute trinitrobenzene sulfonic acid-induced UC. In another study, the inflammatory markers such as IL-17, IL-23, IFN- $\gamma$ and IL- 6 were measured to assess the therapeutic efficacy of human umbilical cord MSCs and were shown to be significantly decreased in the treated mice (41). In the present study, during the follow-up, the levels of cytokines in group I, including TNF- $\alpha$, IL-6 and IFN- $\gamma$, were not significantly changed compared with group II. This may be due to the complexity of the body; however, the exact mechanisms need to be clarified.

Although theexactmechanismsunderlying MSC-mediated suppression of lymphocyte proliferation remain essentially unknown, it is possible that MSCs can accelerate apoptosis of active inflammatory cells. Akiyama et al (42) showed that the systemic infusion of MSC-induced T cell apoptosis via the Fas ligand-dependent Fas pathway, reducing symptoms of dextran sulfate sodium-induced colitis. MSCs modulate their micro-environment by exerting powerful immunosuppressive effects $(43,44)$. These cells inhibit cell proliferation and the cytotoxic potential of NK cells and CD8 ${ }^{+} \mathrm{T}$ cells (45). Furthermore, MSCs impair the maturation, cytokine production and $\mathrm{T}$ cell stimulatory capacity of dendritic cells. In addition, MSCs suppress the proliferation and antibody production of $\mathrm{B}$ cells, inhibit the proliferation and cytokine secretion of $\mathrm{CD}^{+} \mathrm{T}$ lymphocyte subsets, and promote the expansion of regulatory $\mathrm{T}$ cell populations (46). 
In the present clinical trial, there were no evident adverse reactions following $\mathrm{MSC}$ infusion in any of the patients who completed the study protocol, and no chronic side effects or lingering effects appeared during the follow-up period. In addition, the therapeutic effect of MSC infusion was sustained during the entire follow-up period. However, the chronic effect of MSC infusion in treating UC need to explore by an extended follow-up period. In conclusion, MSC infusion may become a useful and safe therapy for patients with UC.

\section{Acknowledgements}

The present study was supported by the Human Umbilical Cord Mesenchymal Stem Cell Bank (Qingdao, China).

\section{References}

1. Chinnadurai R, Ng S, Velu V and Galipeau J: Challenges in animal modelling of mesenchymal stromal cell therapy for inflammatory bowel disease. World J Gastroenterol 21: 4779-4787, 2015.

2. Hiraoka S, Kato J, Moritou Y, Takei D, Inokuchi T, Nakarai A, Takahashi S, Harada K, Okada $\mathrm{H}$ and Yamamoto K: The earliest trough concentration predicts the dose of tacrolimus required for remission induction therapy in ulcerative colitis patients. BMC Gastroenterol 15: 53, 2015.

3. Sameshima S, Koketsu S, Takeshita E, Kubota Y, Okuyama T, Saito K, Ueda Y, Sawada T and Oya M: Surgical resections of ulcerative colitis associated with dysplasia or carcinoma. World J Surg Oncol 13: 70, 2015.

4. Chande N, Tsoulis DJ and MacDonald JK: Azathioprine or 6-mercaptopurine for induction of remission in Crohn's disease. Cochrane Database Syst Rev 30: CD000545, 2013.

5. Naganuma M, Fujii T and Watanabe M: Treatment strategy for refractory inflammatory bowel disease to improve endoscopic lesions and long-term prognosis. Nihon Rinsho Meneki Gakkai Kaishi 35: 99-106, 2012 (In Japanese).

6. Castro-Manrreza ME and Montesinos JJ: Immunoregulation by mesenchymal stem cells: Biological aspects and clinical applications. J Immunol Res 2015: 394917, 2015.

7. Seidelin JB, Coskun M, Kvist PH, Holm TL, Holgersen K and Nielsen OH: IL-33 promotes GATA-3 polarization of gut-derived $\mathrm{T}$ cells in experimental and ulcerative colitis. J Gastroenterol 50: 180-190, 2015.

8. Gordon H, Trier Moller F, Andersen V and Harbord M: Heritability in inflammatory bowel disease: From the first twin study to genome-wide association studies. Inflamm Bowel Dis 21: 1428-1434, 2015.

9. Liu W, Morschauser A, Zhang X, Lu X, Gleason J, He S, Chen HJ, Jankovic V, Ye Q, Labazzo K, et al: Human placenta-derived adherent cells induce tolerogenic immune responses. Clin Transl Immunology 3: e14, 2014.

10. Guo Z, Zhou X, Li J, Meng Q, Cao H, Kang L, Ni Y, Fan H and Liu Z: Mesenchymal stem cells reprogram host macrophages to attenuate obliterative bronchiolitis in murine orthotopic tracheal transplantation. Int Immunopharmacol 15: 726-734, 2013.

11. Huang H, He J, Teng X, Yu Y, Ye W, Hu Y and Shen Z: Combined intrathymic and intravenous injection of mesenchymal stem cells can prolong the survival of rat cardiac allograft associated with decrease in miR-155 expression. J Surg Res 185: 896-903, 2013.

12. Choi EW, Shin IS, Lee HW, Park SY, Park JH, Nam MH, Kim JS, Woo SK, Yoon EJ, Kang SK, et al: Transplantation of CTLA4Ig gene-transduced adipose tissue- derived mesenchymal stem cells reduces inflammatory immune response and improves Th1/Th2 balance in experimental autoimmune thyroiditis. J Gene Med 13: 3-16, 2011.

13. Bressler B, Marshall JK, Bernstein CN, Bitton A, Jones J, Leontiadis GI, Panaccione R, Steinhart AH and Tse F: Clinical practice guidelines for the medical management of nonhospitalized ulcerative colitis: The Toronto consensus. Gastroenterology 48: 1035-1058, 2015.
14. Suzuki Y, Uchiyama K, Kato M, Matsuo K, Nakagawa T, Kishikawa H, Kimura N, Kasanuki J and Ino S: Potential utility of a new ulcerative colitis segmental endoscopic index combining disease severity and the extent of inflammation. J Clin Gastroenterol 49: 401-406, 2015.

15. Leong RW, Huang T, Ko Y, Jeon A, Chang J, Kohler F and Kariyawasam V: Prospective validation study of the International Classification of Functioning, Disability and Health scorein Crohn's disease and ulcerative colitis. J Crohns Colitis 8: 1237-1245, 2014.

16. Travis SP, Schnell D, Feagan BG, Abreu MT, Altman DG, Hanauer SB, Krzeski P, Lichtenstein GR and Marteau PR: The impact of clinical information on the assessment of endoscopic activity: Characteristics of the ulcerative colitis endoscopic index of severity [UCEIS]. J Crohns Colitis 9: 607-616, 2015.

17. Jharap B, Sandborn WJ, Reinisch W, D'Haens G, Robinson AM, Wang W, Huang B, Lazar A, Thakkar RB and Colombel JF: Randomised clinical study: Discrepancies between patient-reported outcomes and endoscopic appearance in moderate to severe ulcerative colitis. Aliment Pharmacol Ther 42: 1082-1092, 2015.

18. Mokarizadeh A, Delirezh N, Morshedi A, Mosayebi G, Farshid AA and Dalir-Naghadeh B: Phenotypic modulation of auto-reactive cells by insertion of tolerogenic molecules via MSC-derived exosomes. Vet Res Forum 3: 257-261, 2012.

19. Jung J, Moon JW, Choi JH, Lee YW, Park SH and Kim GJ: Epigenetic alterations of IL-6/STAT3 signaling by placental stem cells promote hepatic regeneration in a rat model with CCl4-induced liver injury. Int J Stem Cells 8: 79-89, 2015.

20. Qiu XC, Jin H, Zhang RY, Ding Y, Zeng X, Lai BQ, Ling EA, Wu JL and Zeng YS: Donor mesenchymal stem cell-derived neural-like cells transdifferentiate into myelin-forming cells and promote axon regeneration in rat spinal cord transection. Stem Cell Res Ther 6: 105, 2015.

21. Liu Y, Nie L, Zhao H, Zhang W, Zhang YQ, Wang SS and Cheng L: Conserved dopamine neurotrophic factor-transduced mesenchymal stem cells promote axon regeneration and functional recovery of injured sciatic nerve. PLoS One 9: e110993, 2014.

22. Hyatt AJ, Wang D, van Oterendorp C, Fawcett JW and Martin KR: Mesenchymal stromal cells integrate and form longitudinally-aligned layers when delivered to injured spinal cord via a novel fibrin scaffold. Neurosci Lett 569: 12-17, 2014.

23. Gu H, Xiong Z, Yin X, Li B, Mei N, Li G and Wang C: Bone regeneration in a rabbit ulna defect model: Use of allogeneic adipose-derivedstem cells with low immunogenicity. Cell Tissue Res 358: 453-464, 2014.

24. Lu W, Tang Y, Zhang Z, Zhang X, Yao Y, Fu C, Wang X and Ma G: Inhibiting the mobilization of Ly6C (high) monocytes after acute myocardial infarction enhances the efficiency of mesenchymal stromal cell transplantation and curbs myocardial remodeling. Am J Transl Res 7: 587-597, 2015.

25. Gonçalves Fda C, Schneider N, Pinto FO, Meyer FS, Visioli F, Pfaffenseller B, Lopez PL, Passos EP, Cirne-Lima EO, Meurer L and Paz AH: Intravenous vs intraperitoneal mesenchymal stem cells administration: What is the best route for treating experimental colitis? World J Gastroenterol 20: 18228-18239, 2014.

26. Lin Y, Lin L, Wang Q, Jin Y, Zhang Y, Cao Y and Zheng C: Transplantation of human umbilical mesenchymal stem cells attenuates dextran sulfate sodium-induced colitis in mice. Clin Exp Pharmacol Physiol 42: 76-86, 2015.

27. Marquez-Curtis LA and Janowska-Wieczorek A: Enhancing the migration ability of mesenchymal stromal cells by targeting the SDF-1/CXCR4 axis. Biomed Res Int 2013: 561098, 2013.

28. Wu X, Wang W, Meng C, Yang S, Duan D, Xu W, Liu X, Tang $M$ and Wang H: Regulation of differentiation in trabecular bone-derived mesenchymal stem cells by $\mathrm{T}$ cell activation and inflammation. Oncol Rep 30: 2211-2219, 2013.

29. Liu J, Chen J, Liu B, Yang C, Xie D, Zheng X, Xu S, Chen T, Wang L, Zhang Z, et al: Acellular spinal cord scaffold seeded with mesenchymal stem cells promotes long-distance axon regeneration and functional recovery in spinal cord injured rats. J Neurol Sci 325: 127-136, 2013.

30. Liu L, Chiu PW, Lam PK, Poon CC, Lam CC, Ng EK and Lai PB: Effect of local injection of mesenchymal stem cells on healing of sutured gastric perforation in an experimental model. Br J Surg 102: e158-e168, 2015.

31. Yang JX, Zhang N, Wang HW, Gao P, Yang QP and Wen QP: CXCR4 receptor overexpression in mesenchymal stem cells facilitates treatment of acute lung injury in rats. J Biol Chem 290: 1994-2006, 2015. 
32. Chen CP, Chen YY,Huang JP and Wu YH: The effect of conditioned medium derived from human placental multipotent mesenchymal stromal cells on neutrophils: Possible implications for placental infection. Mol Hum Reprod 20: 1117-1125, 2014

33. Chen Z, He X, He X, Chen X, Lin X, Zou Y, Wu X and Lan P. Bone marrow mesenchymal stem cells ameliorate colitis-associated tumorigenesis in mice. Biochem Biophys Res Commun 450: 1402-1408, 2014

34. Strober W and Fuss IJ: Proinflammatory cytokines in the pathogenesis of inflammatory bowel diseases. Gastroenterology 140: 1756-1767, 2011.

35. Yang C, Li J, Lin H, Zhao K and Zheng C: Nasal mucosa derived-mesenchymal stem cells from mice reduce inflammation via modulating immune responses. PLoS One 10: e0118849, 2015.

36. Gregorini M,Bosio F, Rocca C, Corradetti V, Valsania T, PattonieriEF, Esposito P, Bedino G, Collesi C, Libetta C, et al: Mesenchymal stromal cells reset the scatter factor system and cytokine network in experimental kidney transplantation. BMC Immunol 15: 44, 2014.

37. Chatterjee D, Marquardt N, Tufa DM, Hatlapatka T, Hass R, Kasper C, von Kaisenberg C, Schmidt RE and Jacobs R: Human umbilical cord-derived mesenchymal stem cells utilize activin-A to suppress interferon- $\gamma$ production by natural killer cells. Front Immunol 5: 662, 2014

38. Crucitti A, Corbi M, Tomaiuolo PM, Fanali C, Mazzari A, Lucchetti D, Migaldi M and Sgambato A: Laparoscopic surgery for colorectal cancer is not associated with an increase in the circulating levels of several inflammation-related factors. Cancer Biol Ther 16: 671-677, 2015.

39. Hengartner NE, Fiedler J, Schrezenmeier H, Huber-Lang M and Brenner RE: Crucial role of IL1beta and C3a in the in vitro-response of multipotent mesenchymal stromal cells to inflammatory mediators of polytrauma. PLoS One 10: e0116772, 2015.
40. Forte D, Ciciarello M, Valerii MC, De Fazio L, Cavazza E, Giordano R, Parazzi V, Lazzari L and Laureti S: Human cord blood-derived platelet lysate enhances the therapeutic activity of adipose-derived mesenchymal stromal cells isolated from Crohn's disease patients in a mouse model of colitis. Stem Cell Res Ther 6: 170, 2015

41. Nam YS, Kim N, Im KI, Lim JY, Lee ES and Cho SG: Negative impact of bone-marrow-derived mesenchymal stem cells on dextran sulfate sodium-induced colitis. World J Gastroenterol 21: 2030-2039, 2015.

42. Akiyama K, Chen C, Wang D, Xu X, Qu C, Yamaza T, Cai T, Chen W, Sun L and Shi S: Mesenchymal-stem-cell-induced immunoregulation involves FAS-ligand-/FAS-mediated T cell apoptosis. Cell Stem Cell 10: 544-555, 2012.

43. Selim AO, Selim SA, Shalaby SM, Mosaad H and Saber T: Neuroprotective effects of placenta-derived mesenchymal stromal cells in a rat model of experimental autoimmune encephalomyelitis. Cytotherapy 18: 1100-1113, 2016.

44. Fayyad-Kazan H, Faour WH, Badran B, Lagneaux L and Najar M: The immunomodulatory properties of human bone marrow-derived mesenchymal stromal cells are defined according to multiple immunobiological criteria. Inflamm Res 65: 501-510, 2016

45. Liu H, Ding J, Wang J, Wang Y, Yang M, Zhang Y, Chang F and Chen X: Remission of collagen-induced arthritis through combination therapy of microfracture and transplantation of thermogel-encapsulated bone marrow mesenchymal stem cells. PLoS One 10: e0120596, 2015.

46. Xu P, Bailey-Bucktrout S, Xi Y, Xu D, Du D, Zhang Q, Xiang W, Liu J, Melton A, Sheppard D, et al: Innate antiviral host defense attenuates TGF- $\beta$ function through IRF3-mediated suppression of Smad signaling. Mol Cell 56: 723-737, 2014. 